\title{
La red de ecoaldeas: repoblación, autogobierno, autogestión y autosuficiencia alimentaria
}

España se enfrenta, desde el punto de vista demográfico, a tres grandes desafíos: el progresivo envejecimiento poblacional, el despoblamiento territorial y los efectos de la población flotante. Independientemente de las iniciativas gubernamentales, se observa también en nuestro país, como en el resto del mundo, un fenómeno del retorno al medio rural desde la ciudad protagonizado por movimientos ciudadanos que critican una sociedad industrial estancada y que buscan en el campo una vida en comunidad con un enfoque sostenible. Aunque minoritaria, la experiencia de las denominadas ecoaldeas enriquece los escenarios y formas de vida en el medio rural, planteando posibles modelos de transición para repoblar el medio rural y de montaña.

Francisca Ruiz Escudero | Centro de Investigación y Formación Agrarias de Cantabria (CIFA)

URL de la contribución <www.iaph.es/revistaph/index.php/revistaph/article/view/4469>

El modelo de desarrollo urbano-industrial, fundamentalmente a partir de la segunda mitad del siglo XX, ha provocado profundos cambios en nuestra sociedad. Uno de esos cambios se ejemplifica en el plano territorial, con la situación demográfica del medio rural. Según Camarero Rioja (1991), será entre los años 1955 y 1965 cuando se produzca el mayor despoblamiento rural, conocido como éxodo rural. Sin embargo, la despoblación es un problema que llega hasta nuestros días y que, en los medios de comunicación, se ha venido en calificar como España vaciada ${ }^{1}$. La situación es de tal envergadura que el Ministerio de Política Territorial y Función Pública del Gobierno de España acordó, a comienzos de 2017, elaborar una Estrategia Nacional frente al Reto Demográfico enmarcada dentro del Foro Nacional de Despoblación. Según el documento Estrategia Nacional Frente al Reto Demográfico: Directrices Generales, el $48,4 \%$ de los municipios españoles está por debajo de $12,5 \mathrm{hab} / \mathrm{km}^{2}$ y, entre 2001 y 2018, ha perdido población el 63\% de los municipios españoles. Para revertir esta realidad, la citada Estrategia establece tres grandes desafíos demográficos en España: el progresivo envejecimiento poblacional, el despoblamiento territorial y los efectos de la población flotante.

Sin embargo, ante este panorama se produce otro proceso paralelo inverso, de menor intensidad, que plantea recomposiciones territoriales a partir de cambios en las actividades socioeconómicas de las zonas rurales: por un lado, una dinámica de retorno al campo y una diversificación del sustrato económico donde lo agrícola deja de ser el eje vertebrador de estos espacios. Así, Nates y Raymond (2007: 44) plantean que "desde mediados de los años 80 con la emergencia de movimientos sociales que critican una sociedad industrial estancada, se opera un revés de sensibilidad hacia el campo". De estas recomposiciones territoriales en el medio rural, va a surgir lo que empezará a denominarse como movimiento neorrural, fenómeno que remite al desplazamiento físico de las personas desde la ciudad al medio rural. El neorruralismo aparece, entonces, como una mezcla de búsqueda de nuevos modelos de vida, protesta social, visiones arcadianas de experiencias comunitarias predecesoras, entre otros aspectos; un movimiento de gentes que, en definitiva, buscaban otra forma de vida en el campo. Este proceso de retorno al campo continúa y evoluciona hasta la actualidad, aunque sigue siendo minoritario, a nivel general. Un ejemplo actual y con repercusión mediática ha sido el proyecto de recuperación del pueblo abandonado de Fraguas, en Guadalajara.

\section{Ecoaldeas: vida en comunidad y sostenibilidad}

En ese marco de retorno al campo, encontramos las ecoaldeas como experiencias que buscan una vida en comunidad con un enfoque sostenible. A pesar de que 


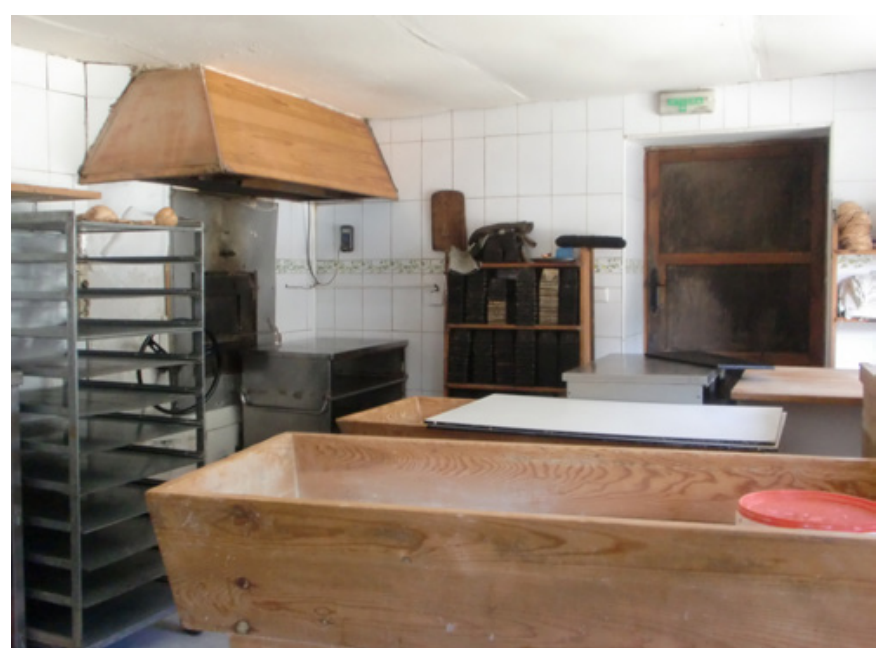

Panadería de la ecoaldea de Lakabe (Navarra) | foto Francisca Ruiz Escudero

se pueden encontrar otros ejemplos comunitarios a lo largo de la historia (ESCORIHUELA, 2008) será en la década de los 80 cuando nazcan la mayoría de este tipo de comunidades ecológicas. El planteamiento de las ecoaldeas deja patente, en su propia etimología, su condición ecológica y el carácter de aldea, entendida como asentamiento humano a pequeña escala. De esta manera, uno de los elementos transversales de dichas experiencias es su apuesta por un modelo de vida comunitaria, es decir, una recuperación del sentido de comunidad. Como reconoce Escorihuela (2000: 79-80) "todas están recorridas por un mismo deseo que las coloca en una igualdad de partida: acabar con el individualismo de la sociedad occidental y buscar formas de vida más participativas, más comunitarias, con mayor integración social y con el entorno, en las que podamos alcanzar un desarrollo pleno como personas, como seres creativos que somos, en las que nos sintamos protegidos en momentos de debilidad y dispuestos a dar en momentos de fuerza. En definitiva, todas las respuestas reinventan a su manera la noción de comunidad". De esta manera, las ecoaldeas son intentos de construir un modelo de vida sostenible, recuperando los lazos de comunidad entre las personas. En la práctica, son proyectos que buscan nuevas formas de relacionarse con el medio a partir de lógicas de autogestión y autosuficiencia (no sólo alimentaria, también en los ámbitos de la construcción, la movilidad, la energía y muchos más; con proyectos de bioconstrucción, huerta, conservas, animales, etc.), buscando modelos cooperativos y/o comunitarios de organización. Un ejemplo es la propia autodefinición de la ecoaldea los Portales "Ios Portales es una comunidad que se fundó en 1984 con la intención de desarrollar formas de vida innovadoras en distintos campos (agricultura orgánica, educación holística, medicinas naturales, arte, energías limpias, economía, desarrollo personal) en progreso continuo hacia una mayor sostenibilidad y autosuficiencia"2.

El concepto de ecoaldeas emerge en los años 80 y proviene del norte de Europa. Del trabajo de Robert y Diane Gilman surgirá la primera definición de ecoaldea "asentamiento humano, concebido a escala humana, que incluye todos los aspectos importantes para la vida, integrándolos respetuosamente en el entorno natural, que apoya formas saludables de desarrollo y que puede persistir indefinidamente" (ESCORIHUELA, 2000: 83). Poco después, en 1995, se organizará una conferencia mundial de ecoaldeas bajo el título Ecoaldeas y comunidades sostenibles: modelos para el siglo $\mathrm{XXI}^{3}$. Dicha conferencia se desarrolló en la comunidad Findhorn (Escocia) y contó con algunos representantes de proyectos ${ }^{4}$ que, a la postre, se convertirán en el germen fundador que crea la Red Global de Ecoaldeas (Global Ecovillage Network, en adelante GEN). Actualmente, encontramos que dicha red se ha expandido y alcanza los cinco continentes, a través de nodos territoriales, a saber: África (GEN África), América Latina (Council of Sustainable Settlements of Latin America), América del Norte (GEN-North America, GENNA), Europa (GEN-Europe) y Asia-Oceanía (Global Ecovillages Network of Oceania and Asia, GENOA). A estos cinco nexos, hay que sumar una vertiente juvenil que se ha creado recientemente, NEXTGEN (Ecovillage Youth Network).

Dada la importancia, cada vez mayor, que las cuestiones de sustentabilidad global tienen a escala planetaria, el GEN se ha configurado como un actor social con cierta repercusión en la esfera pública. Así lo demuestran su estatus consultativo en Naciones Unidas a través del Consejo Económico y Social (ECOSOC), su participación en la Década de Naciones Unidas para el Desarrollo Sostenible 2004-2015 o en la reciente cumbre de París. En 2017, el GEN estuvo investigando, y 


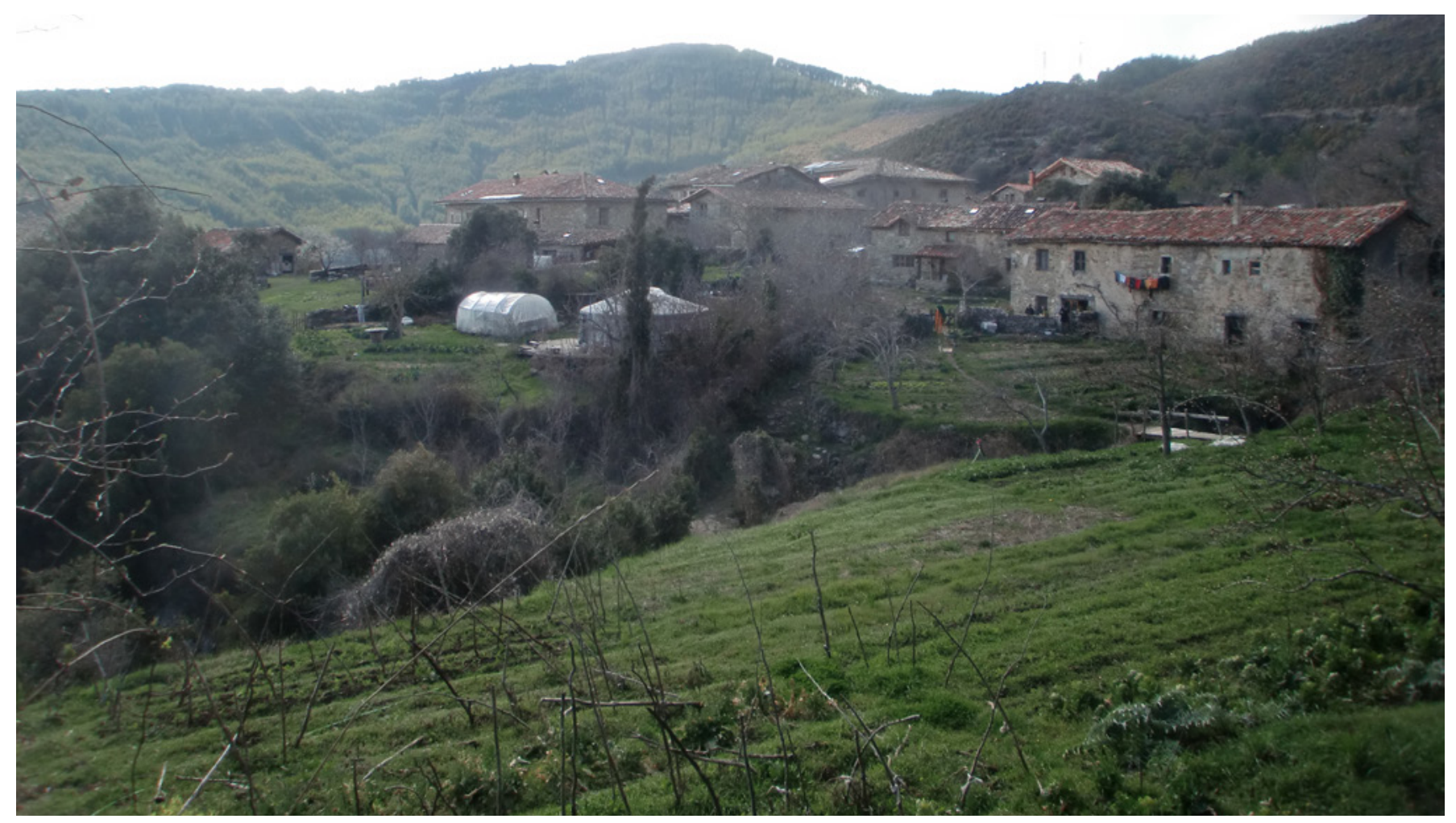

Panorámica de la ecoaldea de Lakabe (Navarra) | foto Francisca Ruiz Escudero

continúa haciéndolo, sobre su contribución a los 17 Objetivos de Desarrollo Sostenible con el estudio de la consecución de estos ODS en 30 ecoaldeas de los 5 continentes. Recientemente, ha participado en la I Conferencia Internacional sobre Despoblación Rural en Navarra (ver nota 8), aportando la visión y experiencias de proyectos ecoaldeanos en todo el mundo. Asimismo y producto de la evolución del propio GEN, el ECOLISE (European Network for Community-Led Initiatives on Climate Change and Sustainability) ha organizado el día europeo de las comunidades sostenibles ${ }^{5}$, el pasado 21 de septiembre de 2019.

Las actividades ${ }^{6}$ del GEN son muy variadas pero se pueden resumir en tres pilares: fomentar el desarrollo de asentamientos humanos sostenibles; favorecer el intercambio de información entre los asentamientos; y difundir información sobre el concepto de ecoaldea y sus valores.

La Red Global de Ecoaldeas está formada por redes de ecoaldeas nacionales y en la península encontra- mos la Red Ibérica de Ecoaldeas ${ }^{7}$ (en adelante, RIE). Tiene su origen en 2001 y, actualmente, está formada por 13 ecoaldeas y otras iniciativas colaboradoras y proyectos miembros. Al igual que el GEN, la RIE trabaja en fomentar el desarrollo de asentamiento sustentables, al tiempo que sirve de plataforma conectando a las diferentes ecoaldeas en la península ibérica. En ese sentido, la RIE ha creado dos proyectos: por un lado, la incubadora de proyectos (propuesta de apoyo al surgimiento de otras comunidades y proyectos sostenibles) $y$, por otro lado, el proyecto Rehabitar ${ }^{8}$ que pretende ser un proyecto piloto que camine hacia el repoblamiento de los pueblos abandonados de Navarra, tomando como referencia la experiencia de las ecoaldeas. Al mismo tiempo, participa de otros proyectos internacionales como el ECOLISE, mencionado anteriormente, SEOC (Sociocracy 3.0 Empowering Organizational Capacities) o Yes to sustainability.

La propuesta de ecoaldea que propone la RIE tiene un andamiaje basado en 4 dimensiones de la sostenibili- 
dad: económica, social, ecológica y cultural. El componente cultural o de visión del mundo viene a enriquecer la óptica clásica del desarrollo sostenible incorporando una perspectiva holística que incluye, entre otras cuestiones, un acercamiento y consciencia de la naturaleza, una cultura de paz y cooperación y una potencial apuesta por la espiritualidad.

Si observamos los proyectos de ecoaldeas en la península, se perciben diferencias en cuanto a la construcción del grupo, la organización interna, la gestión de la economía, las actividades que autogestionan, los trabajos que desarrollan, etc. Así, las ecoaldeas tienen elementos caracterizadores como su visión ecológica y su apuesta por la vida en comunidad como una nueva forma de estar en el planeta, pero no hay una receta única. En la propia RIE se dan cita proyectos distintos, con economías distintas, con organizaciones internas diferentes, con actividades económicas variadas, pero todas tienen una voluntad de cuidar de las personas y del planeta, a partir de nuevas formas de relacionarse y gobernarse. No hay un proyecto tipo, pero sí un anhelo y búsqueda de transformación, de regeneración del planeta, de crear nuevos modelos de gobernanza y relación entre las personas. Una buena forma de acercarse al espacio de las ecoaldeas es acudir a una de las actividades centrales de la RIE, como la organización del encuentro estival (en 2019 se ha celebrado la XXII edición en la ecoaldea de Matavenero, León, entre el 25 y 28 de julio).

En último lugar, cabe señalar que es necesario abordar un análisis, en profundidad, del impacto de estas experiencias comunitarias en el medio rural, por ejemplo, de los cambios producidos en el entorno cercano a dichas ecoaldeas. En este sentido, sería conveniente poder investigar, de forma sistemática, la situación actual de estas iniciativas más allá del punto de vista descriptivo. La aproximación a estas ecoaldeas entraría en los focos de estudio de una nueva sociología de las ausencias y las emergencias (Sousa Santos, 2005), que permita autoevaluar las repercusiones de estas experiencias, como nuevos escenarios de transición en el territorio. Sin embargo, cabe señalar la escasa información, en cuanto a investigación se refiere, sobre estas experiencias, hecho que dificulta un estudio más pormenorizado.

\section{A modo de conclusión}

Se pregunta el sociólogo rural Camarero Rioja (2017) por qué las personas se han quedado en los pueblos ( $y$ no tanto por qué se fueron), en un intento de analizar las motivaciones de las gentes rurales. Respondiendo a esta interrogación y salvando las distancias, se puede señalar que las ecoaldeas serían una propuesta de retorno al campo para quedarse, que plantea una nueva realidad en el territorio, ciertamente poco numerosa, basada en estilos comunitarios y sostenibles de vida rural. Las ecoaldeas se han instalado en el medio rural recuperando o repoblando pueblos porque quieren vivir allí, porque es el medio físico que permite construir modelos de vida sostenible y porque es, desde ese entorno rural, desde donde quieren proyectar su vida a través de la autogestión y autosuficiencia alimentaria. Son "nuevas" experiencias pero que, al mismo tiempo, recuperan viejas dinámicas rurales, entre ellas, el sentido de comunidad o la autogestión comunal de los recursos naturales. Las ecoaldeas, junto con otras iniciativas, enriquecen el escenario de experiencias en el medio rural y se erigen, desde su práctica y su pedagogía cotidiana, como un ejemplo de repoblación del medio rural desde lógicas comunitarias y sostenibles. Son pocas, pero parafraseando al escritor Eduardo Galeano, son un ejemplo que demuestra que actuar sobre la realidad y cambiarla, aunque sea un poquito, es la única manera de probar que la realidad es transformable ${ }^{9}$.

\section{NOTAS}

1. Algunas de las numerosas referencias periodísticas que denotan la actualidad de esta temática son, entre otros, los artículos: "La España vaciada clama por una alianza contra la despoblación, publicado el 31/03/19 <https://elpais.com/sociedad/2019/03/31/actualidad/1554022545_649884.html> [Consulta: 08/08/ 2019]; "La España vacía o la España vaciada", el 10/06/19 [Consulta: 08/08/2019] <https://elpais.com/ elpais/2019/06/06/ideas/1559834099_548373.html>; o incluso referencias a las implicaciones de este despoblamiento con los incendios recientes, en el artículo "Incendios más agresivos por la despoblación de la España va- 
ciada", publicado el 30/06/19 <https://www.elmundo.es/ espana/2019/06/30/5d17ac10fdddffb08e8b460a.html> [Consulta: 08/08/2019].

2. Más información en la ficha de la ecoaldea Los Portales, en <http://rie.ecovillage.org/fileadmin/RIE/Fichas pdf/Ficha_RIE_Portales.pdf> [Consulta: 08/08/2019].

3. Algunos materiales de dicha conferencia se pueden encontrar en la biblioteca de la Agenda Española de Cooperación Internacional para el Desarrollo (AECID) $<$ http://www.aecid.es/galerias/revistas/descargas/repositorio/obrs/folleto-selba-bibliot-coop-00000192.pdf> [Consulta: 02/09/2019].

4. Algunos de los proyectos comunitarios presenten fueron Findhorn (Escocia), The Farm (Tennesee, USA), Lebensgarten (Alemania), Crystal Waters (Australia), Ecoaldea San Petersburgo (Rusia), Gyurufu (Hungria), Proyecto Ladakh (India), Manitou Institute (Colorado, USA) o la Asociación Danesa de Ecoaldeas.

5. Más información en <https://www.sustainable-communities.net/> [Consulta: 08/08/2019].

6. El trabajo del GEN se recoge en sus informes anuales: $<$ https://ecovillage.org/event/european-ecovillage-conference-2019/> [Consulta: 08/08/2019].

7. Para ampliar información sobre la RIE y sus proyectos, ver <http://rie.ecovillage.org/inicio/> [Consulta: 08/08/2019].

8. Más información del Proyecto Rehabitar la tierra desde la sostenibilidad: propuestas para revertir el despoblamiento rural, en el siguiente enlace $<$ https://sites.google. com/site/rehabitarlatierra/home> [Consulta: 08/08/2019].

9. Léase la cita en <http://eduardo-galeano.blogspot. com/2013/07/al-fin-y-al-cabo-somos-lo-que-hacemos. html> [Consulta: 08/08/2019].

\section{BIBLIOGRAFÍA}

- DIAGNÓSTICO estrategia nacional frente al reto demográfico. Eje despoblación [en línea]. Madrid: Ministerio de Política Territorial y Función Pública, 2019, p. 5 <https://www. mptfp.gob.es/dam/es/portal/reto_demografico/Indicadores_ cartografia/Diagnostico_Despoblacion.pdf.pdf> [Consulta: 25/07/2019]

- CAMARERO, L. A. (2017) Trabajadores del campo y familias de la tierra. Instantáneas de la desagrarización. Ager: Revista de estudios sobre despoblación y desarrollo rural, n. ${ }^{\circ} 23,2017$, pp. 163-195

- CAMARERo RIOJA, L. A. (1991) Tendencias recientes y evolución de la población rural en España. Política y Sociedad, n. ${ }^{\circ} 8,1991$, pp. 13-24

- ESCORIHUELA, J. L. (2008) Camino se hace a andar. Del individuo moderno a la comunidad sostenible. Manual para transicioneros. Hornachuelos, Córdoba: Nous, 2008

- ESCORIHUELA, J. L. (coord) (2000) Recuperar el futuro: II Encuentro Estatal de Ecoaldeas. Zaragoza: La Carrucha Cultural, 2000

- estrategia Nacional frente al Reto Demográfico: Directrices Generales [en línea]. Madrid: Ministerio de Política Territorial y Función Pública, $2019<$ https://www. mptfp.gob.es/dam/es/portal/reto_demografico/Estrategia_ Nacional/directrices_generales_estrategia.pdf.pdf> [Consuta: 25/07/2019]

- GEN Europe: Global Ecovillage Network <https://geneurope.org/home/index.htm> [Consulta: 26/07/2019]

- NATES, B.; RAYMOND, S. (2007) Buscando la naturaleza. Migración y dinámicas rurales contemporáneas. Madrid: Anthropos, 2007

- RIE: Red Ibérica de Ecoaldeas <http://rie.ecovillage.org/ inicio/> [Consulta: 26/07/2019]

- SOUSA SANTOS, B. (2005) El milenio huérfano: ensayo para una nueva cultura política. Madrid: Trotta, 2005 